\title{
MULTIPLICITY-FREE HOMOGENEOUS OPERATORS IN THE COWEN-DOUGLAS CLASS
}

\author{
ADAM KORÁNYI AND GADADHAR MISRA
}

\begin{abstract}
In a recent paper, the authors have constructed a large class of operators in the Cowen-Douglas class Cowen-Douglas class of the unit disc $\mathbb{D}$ which are homogeneous with respect to the action of the group Möb - the Möbius group consisting of bi-holomorphic automorphisms of the unit disc $\mathbb{D}$. The associated representation for each of these operators is multiplicity free. Here we give a different independent construction of all homogeneous operators in the Cowen-Douglas class with multiplicity free associated representation and verify that they are exactly the examples constructed previously.
\end{abstract}

The homogeneous operators form a class of bounded operators $T$ on a Hilbert space $\mathcal{H}$. The operator $T$ is said to be homogeneous if its spectrum is contained in the closed unit disc and for every Möbius transformation $g$ the operator $g(T)$, defined via the usual holomorphic functional calculus, is unitarily equivalent to $T$. To every homogeneous irreducible operator $T$ there corresponds an associated unitary representation $\pi$ of the universal covering group $\tilde{G}$ of the Möbius group $G$ :

$$
\pi(\hat{g})^{*} T \pi(\hat{g})=(p \hat{g})(T), \hat{g} \in \tilde{G}
$$

where $p: \tilde{G} \rightarrow G$ is the natural homomorphism. In the paper [6] (see also [3]), it was shown that each homogeneous operator $T$, not necessarily irreducible, in $\mathrm{B}_{m+1}(\mathbb{D})$ admits an associated representation. The representations of $\tilde{G}$ are quite well-known, but we are still far from a complete description of the homogeneous operators. In the recent paper [6], the following theorem was proved.

Theorem 0.1. For any positive real number $\lambda>m / 2, m \in \mathbb{N}$ and an $(m+1)$ - tuple of positive reals $\boldsymbol{\mu}=\left(\mu_{0}, \mu_{1}, \ldots, \mu_{m}\right)$ with $\mu_{0}=1$, there exists a reproducing kernel $K^{(\lambda, \boldsymbol{\mu})}$ on the unit disc such that the adjoint of the multiplication operator $M^{(\lambda, \boldsymbol{\mu})}$ on the corresponding Hilbert space $\mathbf{A}^{(\lambda, \boldsymbol{\mu})}(\mathbb{D})$ is homogeneous. The operators $\left(M^{(\lambda, \boldsymbol{\mu})}\right)^{*}$ are in the Cowen-Douglas class $\mathrm{B}_{m+1}(\mathbb{D})$, irreducible and mutually inequivalent.

In the paper [6], we have presented the operators $M^{(\lambda, \boldsymbol{\mu})}$ in as elementary a way as possible, but this presentation hides the natural ways in which these operators can be found to begin with. Here we will describe another independent construction of the operators $M^{(\lambda, \boldsymbol{\mu})}$. We will also give an exposition of some of the fundamental background material. Finally, we will prove that if $T$ is an irreducible homogeneous operator in $\mathrm{B}_{m+1}(\mathbb{D})$ whose associated representation is multiplicity free then, up to equivalence, $T$ is the adjoint of of the multiplication operator $M^{(\lambda, \boldsymbol{\mu})}$ for some $\lambda>m / 2$ and $\boldsymbol{\mu} \geq 0$.

This research was supported in part by a DST - NSF S\&T Cooperation Programme. 


\section{BACKGROUND MATERIAL}

Although, we intend to discuss homogeneous operators in the Cowen-Douglas class $\mathrm{B}_{n}(\mathbb{D})$, the material below is presented in somewhat greater generality. Here we discuss commuting tuples of operators in the Cowen-Douglas class $\mathrm{B}_{n}(\mathcal{D})$ for some bounded open connected set $\mathcal{D} \subseteq \mathbb{C}^{m}$. The unitary equivalence class of a commuting tuple in $\mathrm{B}_{n}(\mathcal{D})$ is in one to one correspondence with a certain class of holomorphic Hermitian vector bundles (hHvb) on $\mathcal{D}$ [4]. These are distinguished by the property, among others, that the Hermitian structure on the fibre at $w \in \mathcal{D}$ is induced by a reproducing kernel $K$. It is shown in [4] that the corresponding operator can be realized as the adjoint of the commuting tuple multiplication operator $\mathbf{M}$ on the Hilbert space $\mathcal{H}$ of holomorphic functions with reproducing kernel $K$.

Start with a Hilbert space $\mathcal{H}$ of $\mathbb{C}^{n}$ - valued holomorphic functions on a bounded open connected set $\mathcal{D} \subseteq \mathbb{C}^{m}$. Assume that the Hilbert space $\mathcal{H}$ contains the set of vector valued polynomials and that these form a dense subset in $\mathcal{H}$. We also assume that there is a reproducing kernel $K$ for $\mathcal{H}$. We use the notation $K_{w}(z):=K(z, w)$.

Recall that a positive definite kernel $K: \mathcal{D} \times \mathcal{D} \rightarrow \mathbb{C}^{n \times n}$ on $\mathcal{D}$ defines an inner product on the linear span of $\left\{K_{w}(\cdot) \xi: w \in \mathcal{D}, \xi \in \mathbb{C}^{n}\right\} \subseteq \operatorname{Hol}\left(\mathcal{D}, \mathbb{C}^{n}\right)$ by the rule

$$
\left\langle K_{w}(\cdot) \xi, K_{u}(\cdot) \eta\right\rangle=\left\langle K_{w}(u) \xi, \eta\right\rangle, \xi, \eta \in \mathbb{C}^{n} .
$$

(On the right hand side $\langle$,$\rangle denotes the inner product of \mathbb{C}^{n}$. We denote by $\varepsilon_{1}, \ldots, \varepsilon_{n}$ the natural basis of $\mathbb{C}^{n}$.) The completion of this subspace is then a Hilbert space $\mathcal{H}$ of holomorphic functions on $\mathcal{D}$ (cf. [1]) in which the set of vectors $\left\{K_{w}: w \in \mathcal{D}\right\}$ is dense. The kernel $K$ has the reproducing property, that is,

$$
\left\langle f, K_{w} \xi\right\rangle=\langle f(w), \xi\rangle, f \in \mathcal{H}, w \in \mathcal{D}, \xi \in \mathbb{C}^{m} .
$$

Now, for $1 \leq i \leq m$, we have

$$
M_{i}^{*} K_{w} \xi=\bar{w}_{i} K_{w} \xi, w \in \mathcal{D}, \text { where }\left(M_{i} f\right)(z)=z_{i} f(z), f \in \mathcal{H}
$$

and $\left\{K_{w} \varepsilon_{i}\right\}_{i=1}^{n}$ is a basis for $\cap_{i=1}^{m} \operatorname{ker}\left(M_{i}-w_{i}\right)^{*}, w \in \mathcal{D}$.

The joint kernel of the commuting $m$ - tuple $\mathbf{M}^{*}=\left(M_{1}^{*}, \ldots, M_{m}^{*}\right)$, which we assume to be bounded, then has dimension $n$. The map $\sigma_{i}: w \mapsto K_{\bar{w}} \varepsilon_{i}, w \in \overline{\mathcal{D}}, 1 \leq i \leq n$, provides a trivialization of the corresponding bundle $E$ of Cowen - Douglas (cf. [4]). Here $\left.\overline{\mathcal{D}}:=\left\{z \in \mathbb{C}^{m} \mid \bar{z} \in \mathcal{D}\right\}\right)$.

On the other hand, suppose we start with an abstract Hilbert space $\mathcal{H}$ and a $m$-tuple of commuting operators $\mathbf{T}=\left(T_{1}, \ldots, T_{m}\right)$ in the Cowen - Douglas class $\mathrm{B}_{n}(\mathcal{D})$. Then we have a holomorphic Hermitian vector bundle $E$ over $\mathcal{D}$ with the fibre $E_{w}=\cap_{i=1}^{n} \operatorname{ker}\left(T_{i}-w_{i}\right)$ at $w \in \mathcal{D}$. Following [4, one associates to this a reproducing kernel Hilbert space $\hat{\mathcal{H}}$ consisting of holomorphic functions on $\overline{\mathcal{D}}$ as follows. Take a holomorphic trivialization $\sigma_{i}: \mathcal{D} \rightarrow \mathcal{H}$ with $\sigma_{i}(w), 1 \leq i \leq n$, spanning $E_{w}$. For $f \in \mathcal{H}$, define $\hat{f}_{j}(w):=\left\langle f, \sigma_{j}(\bar{w})\right\rangle_{\mathcal{H}}, w \in \overline{\mathcal{D}}$. Set $\langle\hat{f}, \hat{g}\rangle_{\hat{\mathcal{H}}}:=\langle f, g\rangle_{\mathcal{H}}$. The function $K_{w} \varepsilon_{j}:=\widehat{\sigma_{j}(\bar{w})}$ then serves as the reproducing kernel for the Hilbert space $\hat{\mathcal{H}}$. Note that

$$
\begin{aligned}
\left\langle K_{w}(z) \varepsilon_{j}, \varepsilon_{i}\right\rangle_{\mathbb{C}^{n}} & =\left\langle K_{w} \varepsilon_{j}, K_{z} \varepsilon_{i}\right\rangle_{\hat{\mathcal{H}}} \\
& =\left\langle\widehat{\sigma_{j}(\bar{w})}, \widehat{\sigma_{i}(\bar{z})}\right\rangle_{\hat{\mathcal{H}}} \\
& =\left\langle\sigma_{j}(\bar{w}), \sigma_{i}(\bar{z})\right\rangle_{\mathcal{H}}, z, w \in \overline{\mathcal{D}} .
\end{aligned}
$$


If one applies this construction to the case where $\mathcal{H}$ is a Hilbert space of holomorphic functions on $\mathcal{D}$, possesses a reproducing kernel, say $K$, and the operator $\mathbf{M}^{*}$ is in $\mathrm{B}_{n}(\overline{\mathcal{D}})$ then using the trivialization $\sigma_{i}(w)=K_{\bar{w}} \varepsilon_{i}, w \in \overline{\mathcal{D}}$ for the bundle $E$ defined on $\overline{\mathcal{D}}$, the reproducing kernel for $\hat{\mathcal{H}}$ is

$$
\begin{aligned}
\left\langle K_{w}(z) \varepsilon_{j}, \varepsilon_{i}\right\rangle_{\mathbb{C}^{n}} & =\left\langle K_{w} \varepsilon_{j}, K_{z} \varepsilon_{j}\right\rangle_{\mathcal{H}} \\
& =\left\langle\sigma_{j}(\bar{w}), \sigma_{i}(\bar{z})\right\rangle_{\mathcal{H}} \\
& =\left\langle K_{w} \varepsilon_{j}, K_{z} \varepsilon_{i}\right\rangle_{\hat{\mathcal{H}}}, z, w \in \mathcal{D} .
\end{aligned}
$$

Thus $\mathcal{H}=\hat{\mathcal{H}}$.

Let $G$ be a Lie group acting transitively on the domain $\mathcal{D} \subseteq \mathbb{C}^{d}$. Let $G L(n, \mathbb{C})$ denote the set of non-singular $n \times n$ matrices over the complex field $\mathbb{C}$. We start with a multiplier $J$, that is, a smooth family of holomorphic maps $J_{g}: \mathcal{D} \rightarrow \mathbb{C}^{n \times n}$ satisfying the cocycle relation

$$
J_{g h}(z)=J_{h}(z) J_{g}(h \cdot z), \text { for all } g, h \in G, z \in \mathcal{D},
$$

Let $\operatorname{Hol}\left(\mathcal{D}, \mathbb{C}^{n}\right)$ be the linear space consisting of all holomorphic functions on $\mathcal{D}$ taking values in $\mathbb{C}^{n}$. We then obtain a natural (left) action $U$ of the group $G$ on $\operatorname{Hol}\left(\mathcal{D}, \mathbb{C}^{n}\right)$ :

$$
\left(U_{g} f\right)(z)=J_{g^{-1}}(z) f\left(g^{-1} \cdot z\right), f \in \operatorname{Hol}\left(\mathcal{D}, \mathbb{C}^{n}\right), z \in \mathcal{D}
$$

Let $\mathbb{K} \subseteq G$ be the compact subgroup which is the stabilizer of 0 . For $h, k$ in $\mathbb{K}$, we have $J_{k h}(0)=J_{h}(0) J_{k}(0)$ so that $k \mapsto J_{k}(0)^{-1}$ is a representation of $\mathbb{K}$ on $\mathbb{C}^{n}$.

As in [6], we say that if a reproducing kernel $K$ transforms according to the rule

$$
J(g, z) K(g(z), g(\omega)) J(g, \omega)^{*}=K(z, \omega)
$$

for all $g \in \tilde{G} ; z, \omega \in \mathbb{D}$, then $K$ is quasi-invariant.

Proposition 1.1 ([6], Proposition 2.1). Suppose $\mathcal{H}$ has a reproducing kernel $K$. Then $U$ defined by (1.2) is a unitary representation if and only if $K$ is quasi-invariant.

Let $g_{z}$ be an element of $G$ which maps 0 to $z$, that is $g_{z} \cdot 0=z$.

For quasi-invariant $K$ we have

$$
K\left(g_{z} \cdot 0, g_{z} \cdot 0\right)=\left(J_{g_{z}}(0)\right)^{-1} K(0,0)\left(J_{g_{z}}(0)^{*}\right)^{-1},
$$

which shows that $K(z, z)$ is uniquely determined by $K(0,0)$. For each $z$ in $\mathcal{D}$, the positive definite matrix $K(z, z)$ gives the Hermitian structure of our vector bundle.

Given any positive definite matrix $K(0,0)$ such that

$$
J_{k}(0)^{-1} K(0,0)=K(0,0) J_{k}(0)^{*} \text { for all } k \in \mathbb{K},
$$

that is, the inner product $\langle K(0,0) \cdot \mid \cdot\rangle$ is invariant under $J_{k}(0)$, (1.4) defines a Hermitian structure on the homogeneous vector bundle determined by $J_{g}(z)$. In fact, $K(z, z)$, for any $z \in \mathcal{D}$ is well defined, because if $g_{z}^{\prime}$ is another element of $G$ such that $g_{z}^{\prime} \cdot 0=z$ then $g_{z}^{\prime}=g_{z} k$ 
for some $k \in \mathbb{K}$. Hence

$$
\begin{aligned}
K\left(g_{z}^{\prime} \cdot 0, g_{z}^{\prime} \cdot 0\right) & =K\left(g_{z} k \cdot 0, g_{z} k \cdot 0\right) \\
& =\left(J_{g_{z}}(0)\right)^{-1} K(0,0)\left(J_{g_{z} k}(0)^{*}\right)^{-1} \\
& =\left(J_{k}(0) J_{g_{z}}(k \cdot 0)\right)^{-1} K(0,0)\left(J_{g_{z}}(k \cdot 0)^{*} J_{k}(0)^{*}\right)^{-1} \\
& =\left(J_{g_{z}}(0)\right)^{-1}\left(J_{k}(0)\right)^{-1} K(0,0)\left(J_{k}(0)^{*}\right)^{-1}\left(J_{g_{z}}(0)^{*}\right)^{-1} \\
& =\left(J_{g_{z}}(0)\right)^{-1} K(0,0)\left(J_{g_{z}}(0)^{*}\right)^{-1} \\
& =K\left(g_{z} \cdot 0, g_{z} \cdot 0\right)
\end{aligned}
$$

This gives a good overview of all the Hermitian structures of a homogeneous holomorphic vector bundle. But not all such bundles arise from a reproducing kernel. Starting with a positive matrix satisfying (1.5), (1.4) gives us $K(z, z)$, but there is no guarantee (and is false in general) that $K(z, z)$ extends to a positive definite kernel on $\mathcal{D} \times \mathcal{D}$. It is, however, true that if there is such an extension then it is uniquely determined by $K(z, z)$ (because $K(z, w)$ is holomorphic in $z$ and antiholomorphic in $w)$.

This leaves us with the following possible strategy for finding the homogeneous operators in the Cowen - Douglas class. Find all multipliers, (i.e., holomorphic homogeneous vector bundles (hhvb)) such that there exists $K(0,0)$ satisfying (1.5) and consider all such $K(0,0)$. Then determine which of the $K(z, z)$ obtained form (1.4) extends to a positive definite kernel on $\mathcal{D} \times \mathcal{D}$. Then check if the multiplication operator is well-defined and bounded on the corresponding Hilbert space.

Let $\mathcal{H}$ be a Hilbert space consisting of $\mathbb{C}^{n}$ - valued holomorphic functions on some domain $\mathcal{D}$ possessing a reproducing kernel $K$. The sections of the corresponding holomorphic Hermitian vector bundle defined on $\mathcal{D}$ have many different realizations. The connection between two of these is given by a $n \times n$ invertible matrix valued holomorphic function $\varphi$ on $\mathcal{D}$. For $f \in \mathcal{H}$, consider the map $\Gamma_{\varphi}: f \mapsto \tilde{f}$, where $\tilde{f}(z)=\varphi(z) f(z)$. Let $\tilde{\mathcal{H}}=\{\tilde{f}: f \in \mathcal{H}\}$. The requirement that the map $\Gamma_{\varphi}$ is unitary, prescribes a Hilbert space structure for the function space $\tilde{\mathcal{H}}$. The reproducing kernel for $\tilde{\mathcal{H}}$ is easily calculated

$$
\tilde{K}(z, w)=\varphi(z) K(z, w) \varphi(w)^{*} .
$$

It is also easy to verify that $\Gamma_{\varphi} M \Gamma_{\varphi}^{*}$ is the multiplication operator $M: \tilde{f} \mapsto z \tilde{f}$ on the Hilbert space $\tilde{\mathcal{H}}$. Suppose we have a unitary representation $U$ given by a multiplier $J$ acting on $\mathcal{H}$ according to (1.2). Transplanting this action to $\tilde{\mathcal{H}}$ under the isometry $\Gamma_{\varphi}$, it becomes

$$
\left(\tilde{U}_{g^{-1}} \tilde{f}\right)(z)=\tilde{J}_{g}(z) \tilde{f}(g \cdot z)
$$

where the new multiplier $\tilde{J}$ is given in terms of the original multiplier $J$ by

$$
\tilde{J}_{g}(z)=\varphi(z) J_{g}(z) \varphi(g \cdot z)^{-1} .
$$

Of course, now $\tilde{K}$ transforms according to (1.3), with the aid of $\tilde{J}$. If we want, we can now ensure that, by passing from $\mathcal{H}$ to an appropriate $\tilde{\mathcal{H}}, \tilde{K}(z, 0) \equiv 1$. We merely have to set $\varphi(z)=K(0,0)^{1 / 2} K(z, 0)^{-1}$. Thus the reproducing kernel $\tilde{K}$ is almost unique. The only freedom left is to multiply $\varphi(z)$ by a constant unitary $n \times n$ matrix. Once the kernel is normalized, we have

$$
J_{k}(z)=J_{k}(0), z \in \mathbb{D}, k \in \mathbb{K} \text {. }
$$


In fact,

$$
I=K(z, 0)=J_{k}(z) K(k \cdot z, 0) J_{k}(0)^{*}=J_{k}(z) J_{k}(0)^{-1}
$$

and the statement follows. Therefore, once the kernel $K$ is normalized, we have

$$
\left(U_{k^{-1}} f\right)(z)=J_{k}(0) f(k \cdot z), k \in \mathbb{K} .
$$

Given a multiplier $J$, there is always the following method for constructing a Hilbert space with a quasi-invariant Kernel $K$ transforming according to (1.4). We look for a functional Hilbert space possessing this property among the weighted $L^{2}$ spaces of holomorphic functions on $\mathcal{D}$. The norm on such a space is

$$
\|f\|^{2}=\int_{\mathcal{D}} f(z)^{*} Q(z) f(z) d V(z)
$$

with some positive matrix valued function $Q(z)$. Clearly, this Hilbert space possesses a reproducing kernel $K$. The condition that $U_{g^{-1}}$ in (1.2) is unitary is

$$
\begin{array}{r}
\int_{\mathcal{D}} f(g \cdot z)^{*} J_{g}^{*}(z) Q(z) J_{g}(z) f(g \cdot z) d V(z)=\int_{\mathcal{D}} f(w)^{*} Q(w) f(w) d V(w) \\
=\int_{\mathcal{D}} f(g \cdot z)^{*} Q(g \cdot z) f(g \cdot z)\left|\frac{\partial(g \cdot z)}{\partial(z)}\right|^{2} d V(z),
\end{array}
$$

that is,

$$
Q(g \cdot z)=J_{g}(z)^{*} Q(z) J_{g}(z)\left|\frac{\partial(g \cdot z)}{\partial(z)}\right|^{-2}
$$

which is equation (1.3) with $J_{g}(z)$ replaced by $\frac{\partial(g \cdot z)}{\partial(z)} J_{g}(z)^{*-1}$.

Given the multiplier $J_{g}(z), Q(z)$ is again determined by $Q=Q(0)$, and (just as in the case of $K(0,0)=A$ ) it must be a positive matrix commuting with all $J_{k}(0), k \in \mathbb{K}$. (It is assumed that each $J_{k}(0)$ is unitary).

In this way, we can construct many examples of homogeneous operators in $\mathrm{B}_{n}(\mathcal{D})$ but not all.

Even, not all the the homogeneous operators in $\mathrm{B}_{1}(\mathbb{D})$ come from this construction. There is a homogeneous operator in the class $\mathrm{B}_{1}(\mathbb{D})$ corresponding to the multiplier $J(g, z)=$ $\left(g^{\prime}(z)\right)^{\lambda}, \lambda \in \mathbb{R}$ exactly when $\lambda>0$. The reproducing kernel is $K(z, w)=(1-z \bar{w})^{-2 \lambda}$. But such an operator arises from the construction outlined above only if $\lambda \geq 1 / 2$.

Never the less, the homogeneous operators constructed in the manner described above are of interest since they happen to be exactly the subnormal homogeneous operators in this class (cf. [2]).

\section{Computation of the multipliers for the Unit disc}

In the case of $\mathrm{B}_{n}(\mathbb{D})$, it is shown in $[6$ that the bundle corresponding to a homogeneous Cowen-Douglas operator admits an action of the covering group $\tilde{G}$ of the group $G=$ Möb via unitary bundle maps. This suggests the strategy of first finding all the homogeneous holomorphic Hermitian vector bundles (a problem easily solved by known methods) and then determining which of these correspond to an operator in the Cowen-Douglas class. 
We are going to use the method of holomorphic induction. For this, first we describe some basic facts and fix our notation. We follow the notation of [7] which we will use as a reference.

The Lie algebra $\mathfrak{g}$ of $\tilde{G}$ is spanned by $X_{1}=\frac{1}{2}\left(\begin{array}{ll}0 & 1 \\ 1 & 0\end{array}\right), X_{0}=\frac{1}{2}\left(\begin{array}{cc}i & 0 \\ 0 & -i\end{array}\right)$ and $Y=$ $\frac{1}{2}\left(\begin{array}{cc}0 & -i \\ i & 0\end{array}\right)$. The subalgebra $\mathfrak{k}$ corresponding to $\tilde{\mathbb{K}}$ is spanned by $X_{0}$. In the complexified Lie algebra $\mathfrak{g}^{\mathbb{C}}$, we mostly use the complex basis $h, x, y$ given by

$$
\begin{aligned}
& h=-i X_{0}=\frac{1}{2}\left(\begin{array}{cc}
1 & 0 \\
0 & -1
\end{array}\right) \\
& x=X_{1}+i Y=\left(\begin{array}{ll}
0 & 1 \\
0 & 0
\end{array}\right) \\
& y=X_{1}-i Y=\left(\begin{array}{ll}
0 & 0 \\
1 & 0
\end{array}\right)
\end{aligned}
$$

We write $G^{\mathbb{C}}$ for the (simply connected group) $\mathrm{S} L(2, \mathbb{C})$. Let $G_{0}=\mathrm{S} U(1,1)$ be the subgroup corresponding to $\mathfrak{g}$. The group $G^{\mathbb{C}}$ has the closed subgroups $\mathbb{K}^{\mathbb{C}}=\left\{\left(\begin{array}{ll}z & 0 \\ 0 & \frac{1}{z}\end{array}\right)\right.$ : $z \in \mathbb{C}, z \neq 0\}, P^{+}=\left\{\left(\begin{array}{ll}1 & z \\ 0 & 1\end{array}\right): z \in \mathbb{C}\right\}, P^{-}=\left\{\left(\begin{array}{ll}1 & 0 \\ z & 1\end{array}\right): z \in \mathbb{C}\right\} ;$ the corresponding Lie algebras $\mathfrak{k}^{\mathbb{C}}=\left\{\left(\begin{array}{cc}c & 0 \\ 0 & -c\end{array}\right): c \in \mathbb{C}\right\}, \mathfrak{p}^{+}=\left\{\left(\begin{array}{ll}0 & c \\ 0 & 0\end{array}\right): c \in \mathbb{C}\right\}, \mathfrak{p}^{-}=\left\{\left(\begin{array}{ll}0 & 0 \\ c & 0\end{array}\right): c \in \mathbb{C}\right\}$ are spanned by $h, x$ and $y$, respectively. The product $\mathbb{K}^{\mathbb{C}} P^{-}=\left\{\left(\begin{array}{cc}a & 0 \\ b & \frac{1}{a}\end{array}\right): 0 \neq a \in \mathbb{C}, b \in \mathbb{C}\right\}$ is a closed subgroup to be denoted $T$; its Lie algebra is $\mathfrak{t}=\mathbb{C} h+\mathbb{C} y$. The product set $P^{+} \mathbb{K}^{\mathbb{C}} P^{-}=P^{+} T$ is dense open in $G^{\mathbb{C}}$, contains $G$, and the product decomposition of each of its elements is unique. ( $G^{\mathbb{C}} / T$ is the Riemann sphere, $g \tilde{\mathbb{K}} \rightarrow g T,(g \in G)$ is the natural embedding of $\mathbb{D}$ into it.)

According to holomorphic induction [5, Chap 13] the isomorphism classes of homogeneous holomorphic vector bundles are in one to one correspondence with equivalence classes of linear representations $\varrho$ of the pair $(\mathfrak{t}, \tilde{\mathbb{K}})$. Since $\tilde{\mathbb{K}}$ is connected, here this means just the representations of $\mathfrak{t}$. Such a representation is completely determined by the two linear transformations $\varrho(h)$ and $\varrho(y)$ which satisfy the bracket relation of $h$ and $y$, that is,

$$
[\varrho(h), \varrho(y)]=-\varrho(y) .
$$

The $\tilde{G}$-invariant Hermitian structures on the homogeneous holomorphic vector bundle (making it into a homogeneous holomorphic Hermitian vector bundle), if they exist, are given by $\varrho(\tilde{\mathbb{K}})$-invariant inner products on the representation space. An inner product is $\varrho(\tilde{\mathbb{K}})$ invariant if and only if $\varrho(h)$ is diagonal with real diagonal elements in an appropriate basis.

We will be interested only in bundles with a Hermitian structure. So, we will assume without restricting generality, that the representation space of $\varrho$ is $\mathbb{C}^{d}$ and that $\varrho(h)$ is a real diagonal matrix. 
Furthermore, we will be interested only in irreducible homogeneous holomorphic Hermitian vector bundles, this corresponds to $\varrho$ not being the orthogonal direct sum of non-trivial representations. Suppose we have such a $\varrho$; we write $V_{\alpha}$ for the eigenspace of $\varrho(h)$ with eigenvalue $\alpha$. Let $-\eta$ be the largest eigenvalue of $\varrho(h)$ and $m$ be the largest integer such that $-\eta,-(\eta+1), \ldots,-(\eta+m)$ are all eigenvalues. From (2.9) we have $\varrho(y) V_{\alpha} \subseteq V_{\alpha-1}$; this and orthogonality of the eigenspaces imply that $V=\oplus_{j=0}^{m} V_{-(\eta+j)}$ and its orthocomplement are invariant under $\varrho$. So, $V$ is the whole space, and have proved that the eigenvalues of $\varrho(h)$ are $-\eta, \ldots,-(\eta+m)$.

¿From this it is clear that $\varrho$ can be written as the tensor product of the one dimensional representation $\sigma$ given by $\sigma(h)=-\eta, \sigma(y)=0$, and the representation $\varrho^{0}$ given by $\varrho^{0}(h)=$ $\varrho(h)+\eta I, \varrho^{0}(y)=\varrho(y)$. Correspondingly, the bundle for $\varrho$ is the tensor product of a line bundle $L_{\eta}$ and the bundle corresponding to $\varrho^{0}$.

The representation $\varrho^{0}$ has the great advantage that it lifts to a holomorphic representation of the group $T$. It follows that the homogeneous holomorphic vector bundle it determines for $\mathbb{D}, \tilde{G}$, can be obtained as the restriction to $\mathbb{D}$ of the homogeneous holomorphic vector bundle over $G^{\mathbb{C}} / T$ obtained by ordinary induction in the complex analytic category. So, (as a convenient choice) take the local holomorphic cross section $z \mapsto s(z):=\left(\begin{array}{ll}1 & z \\ 0 & 1\end{array}\right)$ of $G^{\mathbb{C}} / T$ over $\mathbb{D}$. In the trivialization given by $s(z)$, the multiplier then appears for $g=\left(\begin{array}{ll}a & b \\ c & d\end{array}\right) \in G^{\mathbb{C}}$ as

$$
\begin{aligned}
J_{g}^{0}(z) & =\varrho^{0}\left(s(z)^{-1} g^{-1} s(g \cdot z)\right) \\
& =\varrho^{0}\left(\begin{array}{cc}
c z+d & 0 \\
-c & (c z+d)^{-1}
\end{array}\right) \\
& =\varrho^{0}\left(\exp \left(\frac{-c}{c z+d} y\right)\right) \varrho^{0}(\exp (2 \log (c z+d) h)) .
\end{aligned}
$$

The last two equalities are simple computations.

For the line bundle $L_{\eta}$, the multiplier is $g^{\prime}(z)^{\eta}$ (we write $g^{\prime}(z)=\frac{\partial g}{\partial z}(z)$ ). Consequently, the multiplier corresponding to the original $\varrho$ is

$$
J_{g}(z)=\left(g^{\prime}(z)\right)^{\eta} J_{g}^{0}(z)
$$

\section{CONDitions IMPOSED BY THE REPRODUCING KERNEL}

We now assume that we have a homogeneous holomorphic vector bundle induced by $\varrho$ as in the preceding sections and that it has a reproducing kernel. Then we derive conditions about the action of $\tilde{G}$ that follow from this hypothesis. In the final section, we will show that these conditions are sufficient: they lead directly to the construction of all homogeneous operators the Cowen-Douglas class with multiplicity free representations.

Under our hypothesis there is a Hilbert space structure on our sections in which the action of $\tilde{G}$ given by (1.4) is unitary. We will study this representation through its $\mathbb{K}$ types (i.e., its restriction to $\tilde{\mathbb{K}}$ ). We first compute the infinitesimal representation. 
For $X \in \mathfrak{g}$, and holomorphic $f$, we have

$$
\begin{aligned}
& \left(U_{X} f\right)(z):=\left(\frac{d}{d t}\right)_{\mid t=0}\left(U_{\exp (t X)} f\right)(z) \\
& \quad=\left(\frac{d}{d t}\right)_{\mid t=0}\left\{\left(\frac{\partial(\exp (-t X) \cdot z)}{\partial z}\right)^{\eta} J_{\exp (-t X)}^{0}(z) f(\exp (-t X) \cdot z)\right\} .
\end{aligned}
$$

There is a local action of $G^{\mathbb{C}}$, so this formula remains meaningful also for $X \in \mathfrak{g}^{\mathbb{C}}$. There are three factors to differentiate. For the last one, $\left(\frac{d}{d t}\right)_{\mid t=0} f(\exp (-t X) \cdot z)=-(X z) f^{\prime}(z)$, and we see that $\exp (t x) \cdot z=\left(\begin{array}{ll}1 & t \\ 0 & 1\end{array}\right) \cdot z=z+t$ gives $x \cdot z=1$; by similar computations, $y \cdot z=-z^{2}, h \cdot z=z$. For the first factor, we interchange the differentiations and get $-\eta \frac{\partial}{\partial z}(X \cdot z)$, i.e., $0,2 \eta z,-\eta$, respectively for $x, y$ and $h$.

To differentiate the factor in the middle, we use its expression (2.10). First for $X=y$, we have

$$
\begin{aligned}
\left.\frac{d}{d t}\right|_{t=0} \varrho^{0}\left(\exp \left(-t(t z+1)^{-1} y\right)\right) & =\left.\frac{d}{d t}\right|_{t=0}\left(\exp \left(-t(t z+1)^{-1} \varrho^{0}(y)\right)\right. \\
& =-\varrho^{0}(y)
\end{aligned}
$$

and

$$
\begin{aligned}
\left.\frac{d}{d t}\right|_{t=0} \varrho^{0}(\exp (2 \log (t z+1) h)) & =\left.\frac{d}{d t}\right|_{t=0} \exp \left(2 \log (t z+1) \varrho^{0}(h)\right) \\
& =2 z \varrho^{0}(h)
\end{aligned}
$$

¿From these, following the conventions of [7] in defining H,E,F, it follows that

$$
\begin{aligned}
(F f)(z):=\left(U_{-y} f\right)(z) & =\left.\frac{d}{d t}\right|_{\mid t=0} J_{\exp (t y)}(z) f(\exp (t y) \cdot z) \\
& =\left(-2 \eta z I+2 z \varrho^{0}(h)-\varrho^{0}(y)\right) f(z)-z^{2} f^{\prime}(z) .
\end{aligned}
$$

Similar, simpler computations give, for $g=\exp (t x)=\left(\begin{array}{cc}1 & t \\ 0 & 1\end{array}\right)$

$$
(E f)(z):=\left(U_{x} f\right)(z)=-f^{\prime}(z) .
$$

Finally, for $g=\exp (t h)=\left(\begin{array}{cc}e^{t / 2} & 0 \\ 0 & e^{-t / 2}\end{array}\right)$, we have

$$
J_{\exp (t h)}(z)=\varrho\left(\begin{array}{cc}
e^{-t / 2} & 0 \\
0 & e^{t / 2}
\end{array}\right)=\exp (-t) \varrho^{0}(h)
$$

Hence it is not hard to verify that

$$
(H f)(z):=\left(U_{h} f\right)(z)=\left(-\eta I+\varrho^{0}(h)\right) f(z)-z f^{\prime}(z)
$$

Under our hypothesis, we have a reproducing kernel and $U$ is unitary. From our computations above, we can determine how $U$ decomposes into irreducibles. The infinitesimal representation of $U$ acts on the vector valued polynomials; a good basis for this space is $\left\{\varepsilon_{j} z^{n}\right.$ : $n \geq 0\} ; \varepsilon_{j}$ is the $j$ th natural basis vector in $\mathbb{C}^{d}$. We have $H\left(\varepsilon_{j} z^{n}\right)=-(\eta+j+n)\left(\varepsilon_{j} z^{n}\right)$, so the lowest $\mathbb{K}$ - types of the irreducible summands are spanned by the $\varepsilon_{j}$. This space is also 
the kernel of $E$. So, $U$ is direct sum of discrete series representations $\left(U^{\eta+j}\right.$, in the notation of [7]), each one appearing as many times as $-(\eta+j)$ appears on the diagonal of $\varrho(h)$.

\section{The multiplicity-FReE CASE}

In order to be able to use the computations of [6] without confusion, we introduce the parameter $\lambda=\eta+\frac{m}{2}$.

From the last remark of the preceding section, it is clear that if $U$ is multiplicity-free then $\varrho(h)$ is an $(m+1) \times(m+1)$ matrix with eigenvalues $-\lambda+\frac{m}{2},-\lambda+\frac{m}{2}-1, \ldots,-\lambda-\frac{m}{2}$. As $\varrho(h) \varepsilon_{j}=-\left(\lambda-\frac{m}{2}+j\right) \varepsilon_{j}$, (2.9) shows that

$$
\varrho(h)\left(\varrho(y) \varepsilon_{j}\right)=-\left(\lambda+\frac{m}{2}+j+1\right) \varrho(y) \varepsilon_{j} \text {, that is, } \varrho(y) \varepsilon_{j}=\text { const } \varepsilon_{j+1} \text {. }
$$

So, $\varrho(y)$ is a lower triangular matrix (with non-zero entries, otherwise we have a reducible bundle). The homogeneous holomorphic vector bundle determines $\varrho(y)$ only up to a conjugacy by a matrix commuting with $\varrho(h)$, that is, a diagonal matrix. So, we can choose the realization of our bundle by applying an appropriate conjugation such that $\varrho(y)=S_{m}$, the triangular matrix whose $(j, j-1)$ element is $j$ for $1 \leq j \leq m$.

By standard representation theory of $\operatorname{SL}(2, \mathbb{R})$, the vectors $(-F)^{n} \varepsilon_{j}$ are orthogonal and the irreducible subspaces $\mathcal{H}^{(j)}$ for $U$ are $\operatorname{span}\left\{(-F)^{n} \varepsilon_{j}: n \geq 0\right\}$ for $0 \leq j \leq m$. There is also precise information about the norms.

Using this, we can construct an orthonormal basis for our representation space.

For any $n \geq 0$, we let $u_{n}^{j}(z)=(-F)^{n} \varepsilon_{j}$.

To proceed further, we need to find the vectors $u_{n}^{j}(z)$ explicitly. This is facilitated by the following Lemma.

LEMMA 4.1. Let $u$ be a vector with $u_{\ell}(z)=u_{\ell} z^{n-\ell}, 0 \leq \ell \leq m$ and $n \geq 0$. We then have

$$
(-F u)_{\ell}(z)=(2 \lambda-m+\ell+n) u_{\ell} z^{n+1-\ell}+\ell u_{\ell-1} z^{n+1-\ell}, 0 \leq \ell \leq m .
$$

Proof. We recall (3.15) that $-(F f)(z)=2 \lambda z f(z)+S_{m} f(z)-2 z D_{m} f(z)+z^{2} f^{\prime}(z)$ for $f \in \mathcal{H}(n)$, where $D_{m}=-\varrho^{0}(h)$ is the diagonal operator with diagonal $\left\{-\frac{m}{2},-\frac{m}{2}+1, \ldots, \frac{m}{2}\right\}$ and $S_{m}$ is the forward weighted shift with weights $1,2, \ldots, m$. Therefore we have

$$
(-F u)_{\ell}(z)=\left(2 \lambda u_{\ell}+\ell u_{\ell-1}-(m-2 \ell) u_{\ell}+(n-\ell) u_{\ell}\right) z^{n+1-\ell}
$$

completing the proof.

Lemma 4.2. For $0 \leq j \leq m$ and $0 \leq \ell \leq m$, we have

$$
u_{n, \ell}^{j}(z)= \begin{cases}0 & \text { if } 0 \leq \ell \leq j-1 \\
\left(\begin{array}{l}
n \\
k
\end{array}\right)(j+1)_{k}(2 \lambda-m+2 j+k)_{n-k} z^{n-k} & \text { if } j \leq \ell \leq m, k=\ell-j,\end{cases}
$$

where $u_{n, \ell}^{j}(z)$ is the scalar valued function at the position $\ell$ of the $\mathbb{C}^{m+1}$ - valued function $u_{n}^{j}(z):=(-F)^{n} \varepsilon_{j}$.

Proof. The proof is by induction on $n$. The vectors $u_{n}^{j}$ are in $\mathcal{H}(n)$ for $0 \leq j \leq m$. For a fixed but arbitrary positive integer $j, 0 \leq j \leq m$, we see that $u_{n, \ell}^{j}(z)$ is 0 if $n<\ell-j$. We have to verify that $\left(-F u_{n}^{j}\right)(z)=u_{n+1}^{j}(z)$. From the previous Lemma, we have

$$
\left(-F u_{n}^{j}\right)_{\ell}(z)=(2 \lambda-m+\ell+n+j) u_{n, \ell^{j}}^{z^{n+j+1-\ell}+\ell u_{n, \ell-1}^{j} z^{n+j+1-\ell},}
$$


where $\left(-F u_{n}^{j}\right)_{\ell}(z)$ is the scalar function at the position $\ell$ of the $\mathbb{C}^{m+1}$ - valued function $\left(-F u_{n}^{j}\right)(z)$. To complete the proof, we note (using $k=\ell-j$ ) that

$$
\begin{aligned}
& \left(-F u_{n}^{j}\right)_{j+k}(z) \\
& =\left(\left(\begin{array}{l}
n \\
k
\end{array}\right)(j+1)_{k}(2 \lambda-m+2 j+k)_{n-k}(2 \lambda-m+2 j+k+n)+\right. \\
& \left.\left(\begin{array}{c}
n \\
k-1
\end{array}\right)(j+1)_{k}(2 \lambda-m+2 j+k-1)_{n-k}\right) z^{n+1-k} \\
& =(j+1)_{k}(2 \lambda-m+2 j+k)_{n-k} \\
& \left(\left(\begin{array}{l}
n \\
k
\end{array}\right)(2 \lambda-m+2 j+k+n)+\left(\begin{array}{c}
n \\
k-1
\end{array}\right)(2 \lambda-m+2 j+k-1)\right) z^{n+1-k} \\
& =(j+1)_{k}(2 \lambda-m+2 j+k)_{n-k} \\
& \left(\left(\begin{array}{c}
n \\
k
\end{array}\right)+\left(\begin{array}{c}
n \\
k-1
\end{array}\right)(2 \lambda-m+2 j+k-1)+(n+1)\left(\begin{array}{l}
n \\
k
\end{array}\right)\right) z^{n+1-k} \\
& =(j+1)_{k}(2 \lambda-m+2 j+k)_{n-k} \\
& \left(\left(\begin{array}{c}
n+1 \\
k
\end{array}\right)(2 \lambda-m+2 j+k-1)+\left(\begin{array}{c}
n+1 \\
k
\end{array}\right)(n-k+1)\right) z^{n+1-k} \\
& =(j+1)_{k}(2 \lambda-m+2 j+k)_{n-k}\left(\left(\begin{array}{c}
n+1 \\
k
\end{array}\right)(2 \lambda-m+2 j+n)\right) z^{n+1-k} \\
& =(j+1)_{k}\left(\left(\begin{array}{c}
n+1 \\
k
\end{array}\right)(2 \lambda-m+2 j+k)_{n+1-k}\right) z^{n+1-k} \\
& =u_{n+1, j+k}^{j}(z)
\end{aligned}
$$

for a fixed but arbitrary $j, 0 \leq j \leq m$ and $k, 0 \leq k \leq m-j$. This completes the proof.

On $\mathcal{H}^{(j)}$, we have the representation $U^{\lambda_{j}}$ acting $(0 \leq j \leq m)$, where $\lambda_{j}=\lambda-\frac{m}{2}+j$. Its lowest $\mathbb{K}$ - type is spanned by $\varepsilon_{j}\left(=u_{0}^{j}\right)$ and $H \varepsilon_{j}=\lambda_{j} \varepsilon_{j}$. By [7, Prop 6.14] we have $\left\|(-F)^{k} \varepsilon_{j}\right\|^{2}=\sigma_{k}^{j}\left\|(-F)^{k-1} \varepsilon_{j}\right\|^{2}$ with

$$
\sigma_{k}^{j}=\left(2 \lambda_{j}+k-1\right) k
$$

for all $k \geq 1$. (Here we used that the constant $q$ in [7, equation (6.33)] equals $\lambda_{j}\left(1-\lambda_{j}\right)$ by [7. Theorem 6.2].) We write

$$
\boldsymbol{\sigma}_{n}^{j}=\prod_{k=1}^{n} \sigma_{k}^{j}
$$

which can be written in a compact form

$$
\boldsymbol{\sigma}_{n}^{j}=\left(\left(2 \lambda_{j}\right)_{n}(1)_{n}\right)
$$

where $(x)_{n}=(x+1) \cdots(x+n-1)$. We stipulate that the binomial co-efficient $\left(\begin{array}{l}n \\ k\end{array}\right)$ as well as $(x)_{n-k}$ are both zero if $n<k$.

The positivity of the normalizing constants $\left(\boldsymbol{\sigma}_{n-j}^{j}\right)^{\frac{1}{2}}(n \geq j)$ is equivalent to the existence of an inner product for which the set of vectors $\mathbf{e}_{n-j}^{j}$ defined by the formula:

$$
\mathbf{e}_{n-j}^{j}=\left(\boldsymbol{\sigma}_{n}^{j}\right)^{-\frac{1}{2}} u_{n-j}^{j}(z), n \geq j, 0 \leq j \leq m
$$

forms an orthonormal set. Of course, the positivity condition is fulfilled if and only if $2 \lambda>m$.

In this way, for fixed $j$, each $e_{n-j}^{j}$ has the same norm for all $n \geq j$. Hence the only possible choice for an orthonormal system is $\left\{\mu_{j} e_{n-j}^{j}: n \geq j\right\}$ for some positive real numbers $\mu_{j}>0$ 
$(0 \leq j \leq m)$. However, we may choose the norm of the first vector, that is, the vector $\mathbf{e}_{0}^{j}$, $0 \leq j \leq m$, arbitrarily. Therefore, all the possible choices for an orthonormal set are

$$
\mu_{j} \mathbf{e}_{n-j}^{j}(z)=\frac{\mu_{j}}{\sqrt{(2 \lambda-m+2 j)_{n-j}} \sqrt{(1)_{n-j}}} u_{n-j}^{j}(z),
$$

$n \geq j, 0 \leq j \leq m$, and $\mu_{j}, 0 \leq j \leq m$ are $m+1$ arbitrary positive numbers.

Let us fix a positive real number $\lambda$ and $m \in \mathbb{N}$ satisfying $2 \lambda>m$. Let $\mathcal{H}^{(\lambda, \boldsymbol{\mu})}$ denote the closed linear span of the vectors $\left\{\mu_{j} e_{n-j}^{j}: 0 \leq j \leq m, n \geq j\right\}$. Then the Hilbert space $\mathcal{H}^{(\lambda, \boldsymbol{\mu})}$ is the representation space for $U$ defined in (1.2). Since the vectors $u_{n}^{j} \perp u_{p}^{k}$ as long as $j \neq k$, it follows that the Hilbert space $\mathcal{H}^{(\lambda, \boldsymbol{\mu})}$ is the orthogonal direct sum $\oplus_{j=0}^{m} \frac{1}{\mu_{j}} \mathcal{H}^{(j)}$.

We proceed to compute the reproducing kernel by using the orthonormal system $\left\{\mu_{j} e_{n-j}^{j}\right.$ : $n \geq j\}, 0 \leq j \leq m$. We point out that for $0 \leq \ell \leq m$, the entry $\mathbf{e}_{n-j}^{\ell, j} z^{n-j}$ at the position $\ell$ of the vector $\mathbf{e}_{n-j}^{j}(z)$ is 0 for $n<\ell$. Consequently, $\mathbf{e}_{n-j}^{j}$ is the zero vector unless $n \geq j$. The set of vectors $\left\{\mu_{j} \mathbf{e}_{n-j}^{j}: 0 \leq j \leq m, n \geq j\right\}$ is orthonormal in the Hilbert space $\mathcal{H}^{(\lambda, \boldsymbol{\mu})}$. We note that

$$
\mathbf{e}_{n-j}^{j}(z)=\left(\left(e_{n-j}^{\ell, j} z^{n-k}\right)\right)_{\ell=0}^{m}
$$

$$
\begin{aligned}
& \left(\mathbf{e}_{n-j}^{j}(z)\right)_{\ell} \\
& \quad= \begin{cases}0, & 0 \leq \ell \leq j-1 \\
\sqrt{\frac{(2 \lambda+2 j-m+k)_{n-j-k}}{(1)_{n-j-k}}} \sqrt{\frac{(n-j-k+1)_{k}}{(2 \lambda+2 j-m)_{k}} \frac{(j+1)_{k}}{(1)_{k}} z^{n-k},} & j \leq \ell \leq m, k=\ell-j .\end{cases}
\end{aligned}
$$

We have under the hypothesis that we have a reproducing kernel Hilbert space on which the representation $U$ is unitary, explicitly determined an orthonormal basis for this space. Now we are able to answer the question of whether this space really exists. For this it is enough to show that $\sum e_{n}(z){\overline{e_{n}(w)}}^{\mathrm{tr}}$ converges pointwise, the sum then represents the reproducing kernel for this Hilbert space. We will sum the series explicitly, and will verify that it gives exactly the kernels constructed in [6]. This will complete the program of this paper by proving that the examples of [6] give all the homogeneous operators in the Cowen-Douglas class whose associated representation is multiplicity free.

To compute the kernel function, it is convenient to set, for any $n \geq 0$,

$$
\begin{aligned}
G(\boldsymbol{\mu}, n, z) & =\left(\begin{array}{cccccc}
\mu_{0} e_{n}^{0,0} z^{n} & \ldots & 0 & \ldots & 0 \\
\vdots & \ldots & \vdots & \ldots & \vdots \\
\mu_{0} e_{n}^{j, 0} z^{n-j} & \ldots & \mu_{j} e_{n-j}^{j, j} z^{n-j} & \ldots & 0 \\
\vdots & \ldots & \vdots & \ldots & \vdots \\
\mu_{0} e_{n}^{m, 0} z^{n-m} & \ldots & \mu_{j} e_{n-j}^{m, j} z^{n-m} & \ldots & \mu_{m} e_{n-m}^{m, m} z^{n-m}
\end{array}\right) \\
& =\left(\begin{array}{ccc}
z^{n} & \ldots & 0 \\
\vdots & \ddots & \vdots \\
0 & \ldots & z^{n-m}
\end{array}\right)\left(\begin{array}{ccccc}
e_{n}^{0,0} & \ldots & 0 & \ldots & 0 \\
\vdots & \ldots & \vdots & \ldots & \vdots \\
e_{n}^{j, 0} & \ldots & e_{n-j}^{j, j} & \ldots & 0 \\
\vdots & \ldots & \vdots & \ldots & \vdots \\
e_{n}^{m, 0} & \ldots & e_{n-j}^{m, j} & \ldots & e_{n-m}^{m, m}
\end{array}\right)\left(\begin{array}{cccc}
\mu_{0} & \ldots & 0 \\
\vdots & \ddots & \vdots \\
0 & \ldots & \mu_{m}
\end{array}\right) \\
& =D_{n}(z) G(n) D(\boldsymbol{\mu}),
\end{aligned}
$$


where $D_{n}(z), D(\boldsymbol{\mu})$ are the two diagonal matrices and $G(n)=\left(e_{n-j}^{\ell, j}\right)_{\ell, j=0}^{m}$ with $\mathbf{e}_{n-j}^{\ell, j}=0$ if $\ell<j$ or if $n<\ell$. The nonzero entries of the lower triangular matrix $G(n)$, using (4.20), are

$$
\begin{aligned}
& G_{j+k, j}(n)=\frac{\left(\begin{array}{c}
n-j \\
k
\end{array}\right)(j+1)_{k}(2 \lambda-m+2 j+k)_{n-j-k}}{\sqrt{(2 \lambda-m+2 j)_{n-j}} \sqrt{(1)_{n-j}}} \\
& =\frac{\sqrt{(2 \lambda-m+2 j+k)_{n-j-k}}}{\frac{(n-j-k+1)_{k}}{\sqrt{(2 \lambda-m+2 j)_{k}}} \frac{(j+1)_{k}}{(1)_{k}}} \\
& =\sqrt{\frac{(2 \lambda-m+2 j+k)_{n-j-k}}{(2 \lambda-m+2 j)_{k}}} \sqrt{\frac{(n-j-k+1)_{k}}{(1)_{n-j-k}}} \frac{(j+1)_{k}}{(1)_{k}}
\end{aligned}
$$

for $0 \leq k \leq m-j$.

Now, we are ready to compute the reproducing kernel $K_{j}$ for the Hilbert space $\mathcal{H}^{(j)}=$ $\operatorname{span}\left\{e_{n-j}^{j}: n \geq j\right\}, 0 \leq j \leq m$. Recall that $K(z, w)=\sum_{n=0}^{\infty} e_{n}(z) e_{n}(w)^{*}$ for any orthonormal basis $e_{n}, n \geq 0$. This ensures that $K$ is a positive definite kernel. For our computations, we will use the particular orthonormal basis $\mathbf{e}_{n-j}^{j}$ as described in (4.19). Since there are $j$ zeros at the top of each of these basis vectors, it follows that $(\ell, p)$ will be 0 if either $\ell<j$ or $p<j$. We will compute $\left(K_{j}(z, w)\right)$, at $(\ell, p)$ for $j \leq \ell, p \leq m$. For $\ell, p$ as above, we have

$$
\begin{aligned}
\left(K_{j}(z, w)\right)_{\ell, p} & =\sum_{n \geq \max (\ell, p)}^{\infty} \mathbf{e}_{n-j, \ell}^{j}(z) \overline{\mathbf{e}_{n-j, p}^{j}(w)} \\
& =\sum_{n \geq \max (\ell, p)}^{\infty} G_{\ell, j}(n) G_{p, j}(n) z^{n-\ell} \bar{w}^{n-p}
\end{aligned}
$$

We first simplify the co-efficient $G_{\ell, j}(n) G_{p, j}(n)$ of $z^{n-\ell} \bar{w}^{n-p}$. The values of $G_{\ell, j}(n)$ are given in (4.22). Therefore, we have

$$
\begin{aligned}
G_{\ell, j}(n) G_{p, j}(n) \\
=\quad\left(\frac{\left(2 \lambda_{j}+\ell-j\right)_{n-\ell}}{\left(2 \lambda_{j}\right)_{\ell-j}} \frac{(n-\ell+1)_{\ell-j}}{(1)_{n-\ell}} \frac{\left(2 \lambda_{j}+p-j\right)_{n-p}}{\left(2 \lambda_{j}\right)_{p-j}} \frac{(n-\ell+1)_{\ell-j}}{(1)_{n-p}}\right)^{1 / 2} \\
\quad \times \frac{(j+1)_{\ell-j}}{(1)_{\ell-j}} \frac{(j+1)_{p-j}}{(1)_{p-j}} \\
=\frac{\left(2 \lambda_{j}+p-j\right)_{n-p}(n-\ell+1)_{\ell-j}}{\left(2 \lambda_{j}\right)_{\ell-j}(1)_{n-p}}\left(\frac{\left(2 \lambda_{j}+\ell-j\right)_{p-\ell}(n-p+1)_{p-\ell}}{\left(2 \lambda_{j}+\ell-j\right)_{p-\ell}(n-p+1)_{p-\ell}}\right)^{1 / 2} \\
\quad \times \frac{(j+1)_{\ell-j}}{(1)_{\ell-j}} \frac{(j+1)_{p-j}}{(1)_{p-j}} \\
=\frac{\left(2 \lambda_{j}\right)_{p-j}\left(2 \lambda_{j}+p-j\right)_{n-p}(n-\ell+1)_{\ell-j}(n-p+1)_{p-j}}{\left(2 \lambda_{j}\right)_{p-j}\left(2 \lambda_{j}\right)_{\ell-j}(1)_{n-p}(n-p+1)_{\ell-j}} \frac{(j+1}{(1)_{\ell-j}} \frac{(j+1)_{p-j}}{(1)_{p-j}} \\
=\frac{\left(2 \lambda_{j}\right)_{n-j}(n-\ell+1)_{\ell-j}(n-p+1)_{p-j}}{\left(2 \lambda_{j}\right)_{p-j}\left(2 \lambda_{j}\right)_{\ell-j}(1)_{n-j}} \frac{(j+1)_{\ell-j}}{(1)_{\ell-j}} \frac{(j+1)_{p-j}}{(1)_{p-j}} .
\end{aligned}
$$

TheOREM 4.1. Given an arbitrary set $\mu_{0}, \ldots, \mu_{m}$ of positive numbers, and $2 \lambda>m$, we have

$$
K^{(\lambda, \boldsymbol{\mu})}(z, w)=\sum_{j=0}^{m} \mu_{j}^{2} K_{j}(z, w)=\mathbf{B}^{(\lambda, \boldsymbol{\mu})}(z, w) .
$$

As a result, the two Hilbert spaces $\mathcal{H}^{(\lambda, \boldsymbol{\mu})}$ and $\mathbf{A}^{(\lambda, \boldsymbol{\mu})}$ of [6] are equal. 
Proof. We now compare the co-efficients $\left(K_{j}(z, w)\right)_{\ell, p}$ with that of a known Kernel. Let $B^{\lambda_{j}}(z, w)=(1-z \bar{w})^{-2 \lambda_{j}}$, where $B(z, w)=(1-z \bar{w})^{-2}$ is the Bergman kernel on the unit disc. We let $\partial$ and $\bar{\partial}$ denote differentiation with respect to $z$ and $\bar{w}$ respectively. Put

$$
\tilde{\mathbf{B}}^{\left(\lambda_{j}\right)}(z, w)=\left(\partial^{\ell-j} \bar{\partial}^{p-j}(1-z \bar{w})^{-2 \lambda_{j}}\right)_{j \leq \ell, p \leq m} .
$$

We expand the entry at the position $(\ell, p)$ of $\tilde{\mathbf{B}}^{\left(\lambda_{j}\right)}(z, w)$ to see that

$$
\begin{aligned}
& \left(\tilde{\mathbf{B}}^{\left(\lambda_{j}\right)}(z, w)\right)_{\ell, p} \\
& =\sum_{\nu \geq \max (\ell-j, p-j)} \frac{\left(2 \lambda_{j}\right)_{\nu}}{(1)_{\nu}}(\nu-\ell+j+1)_{\ell-j}(\nu+j-p+1)_{p-j} z^{\nu-(\ell-j)} \bar{w}^{\nu-(p-j)} \\
& =\sum_{n \geq \max (\ell, p)} \frac{\left(2 \lambda_{j}\right)_{n-j}}{(1)_{n-j}}(n-\ell+1)_{\ell-j}(n-p+1)_{p-j} z^{n-\ell} \bar{w}^{n-p},
\end{aligned}
$$

where we have set $n=m+j$. Comparing these coefficients with that of $G_{\ell, j}(n) G_{p, j}(n)$, we find that

$$
K_{j}(z, w)=D_{j} \tilde{\mathbf{B}}^{\left(\lambda_{j}\right)}(z, w) D_{j},
$$

where $D_{j}$ is a diagonal matrix with $\frac{1}{\left(2 \lambda_{j}\right)_{\ell-j}} \frac{(j+1)_{\ell-j}}{(1)_{\ell-j}}$ at the $(\ell, \ell)$ position with $j \leq \ell \leq m$. Hence $K_{j}(z, w)=\mathbf{B}^{\left(\lambda_{j}\right)}(z, w)$ which was defined in the equation ([6, equation (4.3)]).

Clearly, we can add up the kernels $K_{j}$ to obtain the kernel $K^{(\lambda, \boldsymbol{\mu})}$ for the Hilbert space $\mathcal{H}^{(\lambda, \boldsymbol{\mu})}=\oplus_{j=0}^{m} \frac{1}{\mu_{j}} \mathcal{H}^{(j)}$. Hence the proof of the theorem is complete.

Corollary 4.1. The irreducible homogeneous operators in the Cowen-Douglas class whose associated representation is multiplicity free are exactly the adjoints of $M^{(\lambda, \boldsymbol{\mu})}$ constructed in [6].

Proof. In our discussion up to here we proved that the Hilbert space $\mathcal{H}^{(\lambda, \boldsymbol{\mu})}$ corresponding to a homogeneous operator in the Cowen - Douglas class has a reproducing kernel given by $K^{(\lambda, \boldsymbol{\mu})}=\sum_{0}^{m} \mu_{j}^{2} K_{j}, 2 \lambda>1, \mu_{1}, \ldots, \mu_{m}>0$. It follows from the Theorem that the kernels obtained this way are the same as (are equivalent to) the kernels constructed in [6]. These operators were shown to be irreducible [6].

We now consider the action of the multiplication operator $M^{(\lambda, \boldsymbol{\mu})}$ on the Hilbert space $\mathcal{H}^{(\lambda, \boldsymbol{\mu})}$. Let $\mathcal{H}(n)$ be the linear span of the vectors

$$
\left\{\mathbf{e}_{n}^{0}(z), \ldots, \mathbf{e}_{n-j}^{j}(z), \ldots, \mathbf{e}_{n-m}^{m}(z)\right\},
$$

where as before, for $0 \leq \ell \leq m, \mathbf{e}_{n-\ell}^{j}(z)$ is zero if $n-\ell<0$. Clearly, $\mathcal{H}^{(\lambda, \boldsymbol{\mu})}=\oplus_{n=0}^{\infty} \mathcal{H}(n)$. We have

$$
\begin{aligned}
z G(n, z) & =D_{n}(z) G(n) D(\boldsymbol{\mu}) \\
& =D_{n+1}(z) G(n) D(\boldsymbol{\mu}) \\
& =D_{n+1}(z) G(n+1) D(\boldsymbol{\mu})\left(D(\boldsymbol{\mu})^{-1} G(n+1)^{-1} G(n) D(\boldsymbol{\mu})\right) .
\end{aligned}
$$

If we let $W(n)=D(\boldsymbol{\mu})^{-1} G(n+1)^{-1} G(n) D(\boldsymbol{\mu})$, then we see that $z \mathbf{e}_{n-j}^{j}(z)=G(\boldsymbol{\mu}, n+$ $1, z) W_{j}(n)$, where $W_{j}(n)$ is the $j$ th column of the matrix $W(n)$. It follows that the operator $M^{(\lambda, \boldsymbol{\mu})}$ defines a block shift $W$ on the representation space $\mathcal{H}^{(\lambda, \boldsymbol{\mu})}$. The block shift $W$ is defined by the requirement that $W: \mathcal{H}(n) \rightarrow \mathcal{H}(n+1)$ and $W_{\mid \mathcal{H}(n)}=W_{n}^{\mathrm{t} r}$. 
Here, we have a construction of the representation space $\mathcal{H}^{(\lambda, \boldsymbol{\mu})}$ along with the matrix representation of the operator $M^{(\lambda, \boldsymbol{\mu})}$ which is independent of the corresponding results from [6].

\section{EXAMPLES}

Recall that $G(\boldsymbol{\mu}, n, z)=D_{n}(z) G(n) D(\boldsymbol{\mu})$. Once we determine the matrix $G(n)$ explicitly, we can calculate both the block weighted shift and the kernel function.

We discuss these calculations in the particular case of $m=1$. First, it is easily seen that

$$
G(n)=\left(\begin{array}{cc}
\left(\frac{(2 \lambda-1)_{n}}{(1)_{n}}\right)^{1 / 2} & 0 \\
\left(\frac{n}{2 \lambda-1}\right)^{1 / 2}\left(\frac{(2 \lambda)_{n-1}}{(1)_{n-1}}\right)^{1 / 2} & \left(\frac{(2 \lambda+1)_{n-1}}{(1)_{n-1}}\right)^{1 / 2}
\end{array}\right) .
$$

The block $W_{n}$ of the weighted shift $W$ is

$$
W_{n}=\left(\begin{array}{cc}
\left(\frac{n+1}{2 \lambda+n-1}\right)^{1 / 2} & 0 \\
-\frac{1}{\mu_{1}}\left(\frac{2 \lambda}{2 \lambda-1}\right)^{1 / 2}\left(\frac{1}{(2 \lambda+n-1)(2 \lambda+n)}\right)^{1 / 2} & \left(\frac{n}{2 \lambda+n}\right)^{1 / 2}
\end{array}\right) .
$$

Finally, the reproducing kernel $K^{(\lambda, \boldsymbol{\mu})}$ with $m=1$ is easily calculated:

$$
K^{(\lambda, \boldsymbol{\mu})}(z, w)=\left(\begin{array}{cc}
\frac{1}{(1-\bar{w} z)^{2 \lambda-1}} & \frac{z}{(1-\bar{w} z)^{2 \lambda}} \\
\frac{\bar{w}}{(1-\bar{w} z)^{2 \lambda}} & \frac{1}{2 \lambda-1} \frac{1+(2 \lambda-1) \bar{w} z}{(1-\bar{w} z)^{2 \lambda+1}}
\end{array}\right)+\mu_{1}^{2}\left(\begin{array}{cc}
0 & 0 \\
0 & \frac{1}{(1-\bar{w} z)^{2 \lambda+1}}
\end{array}\right) .
$$

One might continue the explicit calculations, as above, in the particular case of $m=2$ as well. We begin with the matrix

$$
G(n)=\left(\begin{array}{ccc}
\left(\frac{(2 \lambda-2)_{n}}{(1)_{n}}\right)^{1 / 2} & 0 & 0 \\
\left(\frac{n}{2 \lambda-2}\right)^{1 / 2}\left(\frac{(2 \lambda-1)_{n-1}}{(1)_{n-1}}\right)^{1 / 2} & \left(\frac{(2 \lambda)_{n-1}}{(1)_{n-1}}\right)^{1 / 2} & 0 \\
\left(\frac{n(n-1)}{(2 \lambda-2)(2 \lambda-1)}\right)^{1 / 2}\left(\frac{(2 \lambda)_{n-2}}{(1)_{n-2}}\right)^{1 / 2} & 2\left(\frac{n-1}{2 \lambda}\right)^{1 / 2}\left(\frac{(2 \lambda+1)_{n-2}}{(1)_{n-2}}\right)^{1 / 2} & \left(\frac{(2 \lambda+2)_{n-2}}{(1)_{n-2}}\right)^{1 / 2}
\end{array}\right) .
$$

The block $W_{n}$ of the weighted shift $W$, in this case, is

$$
\left(\begin{array}{ccc}
\left(\frac{n+1}{2 \lambda+n-2}\right)^{1 / 2} & 0 & 0 \\
\frac{-1}{\mu_{1}}\left(\frac{2 \lambda-1}{2 \lambda-2}\right)^{1 / 2}\left(\frac{1}{(2 \lambda+n-1)(2 \lambda+n-2)}\right)^{1 / 2} & \left(\frac{n}{2 \lambda+n-1}\right)^{1 / 2} & 0 \\
\frac{-2}{\mu_{2}}\left(\frac{2 \lambda+1}{(2 \lambda-2)_{3}}\right)^{1 / 2}\left(\frac{n}{(2 \lambda+n-2)_{3}}\right)^{1 / 2} & \frac{-2 \mu_{1}}{\mu_{2}}\left(\frac{2 \lambda+1}{2 \lambda}\right)^{1 / 2}\left(\frac{1}{(2 \lambda+n-1)(2 \lambda+n)}\right)^{1 / 2} & \left(\frac{n-1}{2 \lambda+n}\right)^{1 / 2}
\end{array}\right) .
$$

Finally, the reproducing kernel $K^{(\lambda, \boldsymbol{\mu})}$ with $m=2$ has the form:

$$
\begin{aligned}
K^{(\lambda, \boldsymbol{\mu})}(z, w)= & \left(\begin{array}{ccc}
\frac{1}{(1-\bar{w} z)^{2 \lambda-2}} & \frac{z}{(1-\bar{w} z)^{2 \lambda-1}} & \frac{z^{2}}{(1-\bar{w} z)^{2 \lambda}} \\
\frac{\bar{w}}{(1-\bar{w} z)^{2 \lambda-1}} & \frac{1+(2 \lambda-2) \bar{w} z}{(2 \lambda-2)(1-\bar{w} z)^{2 \lambda}} & \frac{z(2+(2 \lambda-2) \bar{w} z)}{(2 \lambda-2)(1-\bar{w} z)^{2 \lambda+1}} \\
\frac{\bar{w}^{2}}{(1-\bar{w} z)^{2 \lambda}} & \frac{\bar{w}(2+(2 \lambda-2) \bar{w} z)}{(2 \lambda-2)(1-\bar{w} z)^{2 \lambda+1}} & \frac{2+4(2 \lambda-1) \bar{w} z+(2 \lambda-1)(2 \lambda-2) z^{2} \bar{w}^{2}}{(2 \lambda-1)(2 \lambda-2)(1-\bar{w} z)^{2 \lambda+2}}
\end{array}\right) \\
& +\mu_{1}^{2}\left(\begin{array}{ccc}
0 & 0 & 0 \\
0 & \frac{1}{\left(1-\bar{w} z z^{2 \lambda}\right.} & 2 \frac{z}{(1-\bar{w} z)^{2 \lambda+1}} \\
0 & 2 \frac{\bar{w}}{(1-\bar{w} z)^{2 \lambda+1}} & 2 \frac{2}{2 \lambda} \frac{1+2 \lambda \bar{w} z}{(1-\bar{w} z)^{2 \lambda+2}}
\end{array}\right) \\
& +\mu_{2}^{2}\left(\begin{array}{ccc}
0 & 0 & 0 \\
0 & 0 & 0 \\
0 & 0 & \frac{1}{(1-\bar{w} z)^{2 \lambda+2}}
\end{array}\right)
\end{aligned}
$$




\section{REFERENCES}

[1] Aronszajn, N. (1950). Theory of reproducing kernels, Trans. Amer.Math. Soc. 68 337-404.

[2] Bagchi, B. and Misra, G. (1996). Homogeneous tuples of multiplication operators on twisted Bergman spaces. J. Funct. Anal. 136 171-213.

[3] Biswas, I. and Misra, G. (2008). $\widetilde{\mathrm{SL}}(2, \mathbb{R})$ - homogeneous vector bundles. Int. J. Math. 19 1-19.

[4] Cowen, M. J. and Douglas, R. G. (1978). Complex geometry and operator theory. Acta Math. 141 187-261.

[5] Kirilov, A. (1976). Elements of the theory of representations. Springer-Verlag.

[6] Korányi, A. and Misra, G. (2008). Homogeneous operators on Hilbert spaces of holomorphic functions. J. Func. Anal. 254 2419-2436.

[7] Sugiura, M. (1975). Unitary representations and harmonic analysis. An introduction. Kodansha Ltd., Tokyo; Halstead Press [John Wiley \& Sons], New York-London-Sydney.

Lehman College, The City University of New York, Bronx, Ny 10468

E-mail address: adam.koranyi@lehman.cuny.edu

Department of Mathematics, Indian Institute of Science, Bangalore 560012

E-mail address: gm@math.iisc.ernet.in 